\title{
The Evolution of Drug Resistance in Candida
}

\author{
Dr. Anisa LITI \\ “Aleksandër Moisiu” University, Durrës, Faculty of Professional Science, Department of Medicine
}

\begin{abstract}
The epidemiology of Candida infections has changed in recent years. Although Candida albicans is still the main cause of invasive candidiasis in most clinical settings. The use of antifungal drugs in the therapy of fungal diseases can lead to the development of antifungal resistance. A better understanding of the mechanisms and clinical impact of antifungal drug resistance is essential for the efficient treatment of patients with Candida infection and for improving treatment outcomes. We have analysed 100 cases of positive clinical samples, such as vaginal, gastrointestinal, throat, hair, nail, skin samples. These positive Candida samples were examined for their antimycotic resistance. These samples were collected and examinated in the Laboratory of Microbiology, DRSH, Durrës, Albania. We have used seven different antimycotics: Econazole, Nystatin, Miconazole, Clotrimazole, Ketoconazole, Amphotericin, Fluconazole. It has resulted that C. albicans is more sensitive to Fluconazole and more resistente to Miconazole. So, we can say that the best antimycotic for $C$. albicans infections is Fluconazole.
\end{abstract}

Keywords: Candida albicans, commensal organism, dimorphic yeast, antimycotic resistance.

\section{Introduction}

C. albicans is a fungus that normally lives as a commensally organism in humans. However, $C$. albicans is an opportunistic pathogen and one of the main agents of mucosal and systemic fungal infections (Brown et al., 2012). It is a dimorphic fungus, which can be grown up in two main forms:

1. As a unicellular yeast

2. In fibrous form, which can be in the form of chains built from the merger of long cells, also known as the pseudohyphae or in the form of true hypha, which have the form of pipes with parallel page (Sudbery, 2011 ). Filamentous forms prevail in the areas of primary epithelial infection. Morphological transformation may facilitate the introduction of $C$. albicans in the blood and therefore spreads in the form of a systemic infection (Gow et al., 2012). This morphological transformation is essential for the pathogenicity of $C$. albicans (Lo et al., 1997). Although antimycotics used in clinical treatments are various, only a few classes of them are able to treat the mucosal or systemic infections caused by Candida spp. Figure 1.1 shows the action mechanism of the main classes of antimycotics (Spampinato et al., 2013).

In the table below are presented the main classes of antimycotics, their way of action and key members of each class (Spampinato et al., 2013).

Resistance of fungi has to do with their non sensitivity towards the antifungal agents proved through in vitro tests. Microbiological resistance can be primary (innate) or secondary (acquired). Resistance primary is seen in fungus which were not previously exposed to antimycotics, while subsidiary resistance is seen in strains that were previously exposed to antimycotics and is dependent on the change of gene expression (Kanafani et al., 2008). 


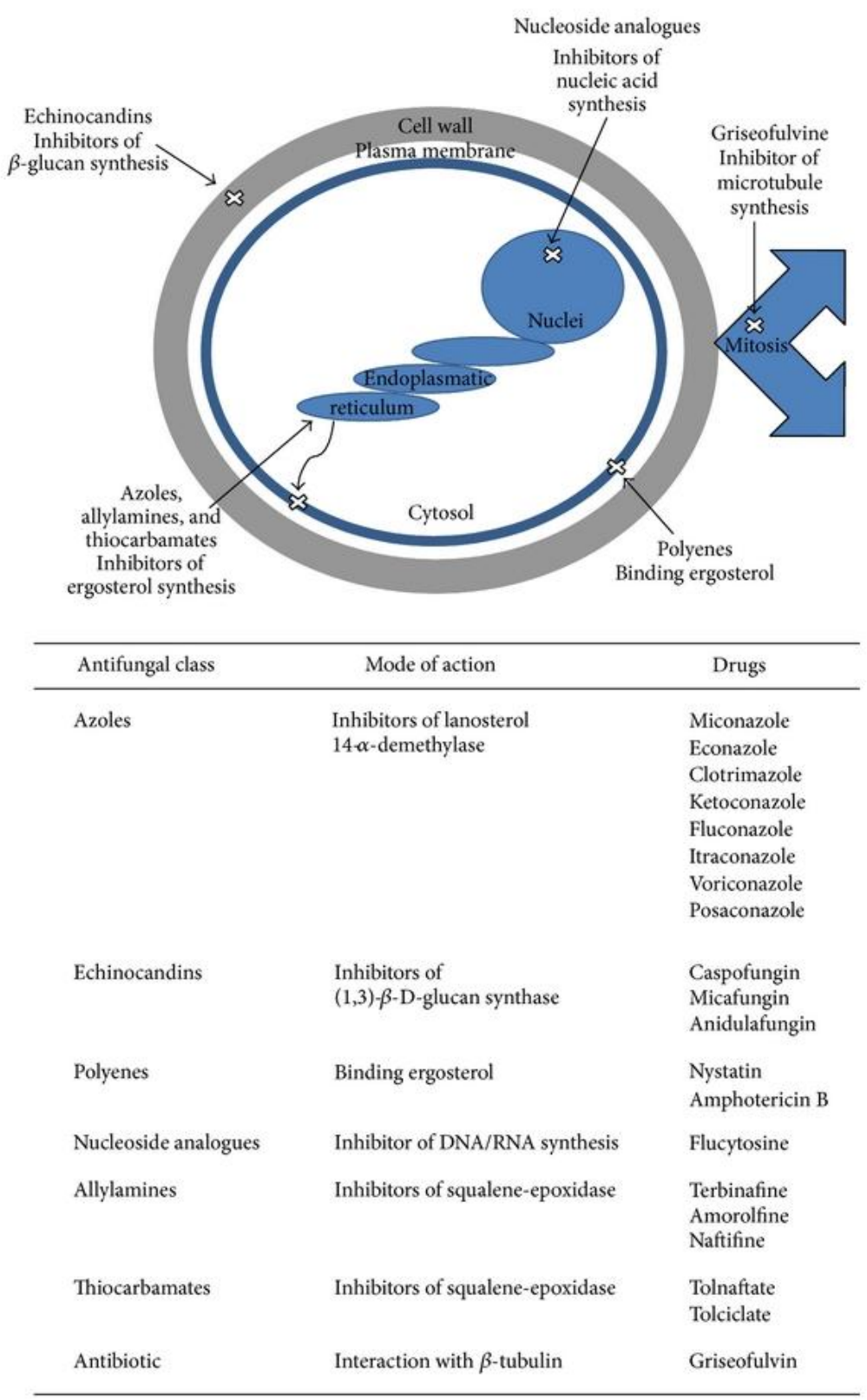

Figure 1.1 Primary targets and mode of action of several antifungal agents.

\section{Materials and methods}

Our study was conducted for the period April 2012-September 2015, in the Laboratory of Microbiology at the Regional Health Directorate, Durres. Initially we have conducted the sampling, isolation and identification of $C$. albicans pathogens in the mouth cavity, urogenital tract, gastrointestinal tract, skin, hair, scalp, nails. To see the impact of various antimycotics on the organism of the study, C. albicans, we have realized the antimycogram of 100 positive cases. For this study we have used seven antimycotics: Ekonazol, Nistatin, Mikonazol, Clotrimazole, Ketonazol, Amphotericin, Fluconazole. Initially we got through a colony of C. albicans from the sample culture, which tested positive, and we planted with marking across the surface of the Petri Plate with Sabouraud Dextrose Agar (SDA). On the surface of the platter we have established a small paper disc of each of the used antimycotics. We have incubated for 24-48 hours. Later, thanks to the diameter of the growth inhibition of pathogenic colony, we determine which of these antimycotics has better devastating action towards pathogen fungus $C$. albicans. At the end was completed the antimycogram sheet, which contains the following information: name and surname of the patient, type of material, the result, the action of each of aforementioned antimycotics (R-resistant towards the used antimycotics, S-sensitive to antimycotics, MS - halfsensitive to antimycotics). 


\section{Results And Discussion}

Towards ekonazol the strains of pathogenic C. albicans fungus resulted sensitive in $26 \%$ of analyzed cases, to nistatin and mikonazol in any case or $0 \%$, to klotrimazol in $50 \%$ of cases, toward ketonazol in $34 \%$ of cases, toward amphotericin in $10 \%$ of cases and to fluconazole in $74 \%$ of cases. So, we see that the C. albicans strains are more sensitive to fluconazole. Resistant to ekonazol these strains resulted in $66 \%$ of cases, to nistatin in $94 \%$ of cases, to mikonazol in all cases, to klotrimazol in $50 \%$ of cases, to ketonazol in $51 \%$ of cases, to amphotericin in $90 \%$ of cases and to fluconazole in $26 \%$ of cases. So, from these antimycogram results, is seen that from these 7 analyzed antimycotics, fluconazole has the highest action against the strains of the studied pathogenic fungus, $C$. albicans. While, the antimycotics on whose the strains of $C$. albicans are more resistant is mikonazol. These results are presented in Figure 1.3.

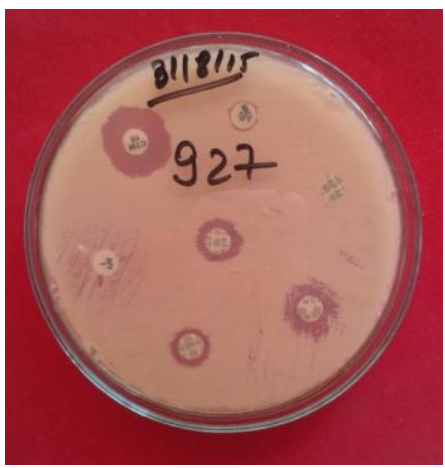

Figure 1.2 Candida albicans antimycogram

This achieved result is confirmed also by literature, where in the study conducted by Jacqueline et al., 2010, was demonstrated that fluconazole is more useful against candidiasis in adults and children. The treatment through fluconazole is $90 \%$ effective and easily distributed throughout the body. In a study conducted in 2013 in the district of Elbasan, where it is analyzed also the antimycotic action of $C$. albicans strains, it showed that $C$. albicans is resistant to amphotericin and klotrimazol. While the antimycotic that fight it better, and against whom C. albicans is sensitive, resulted nistatin. C. albicans in our study is quite resistant toward nistatin and in no case resulted sensitive to it. This can be explained by the fact that the strains that we examined may have acquired resistance to this antimycotics.

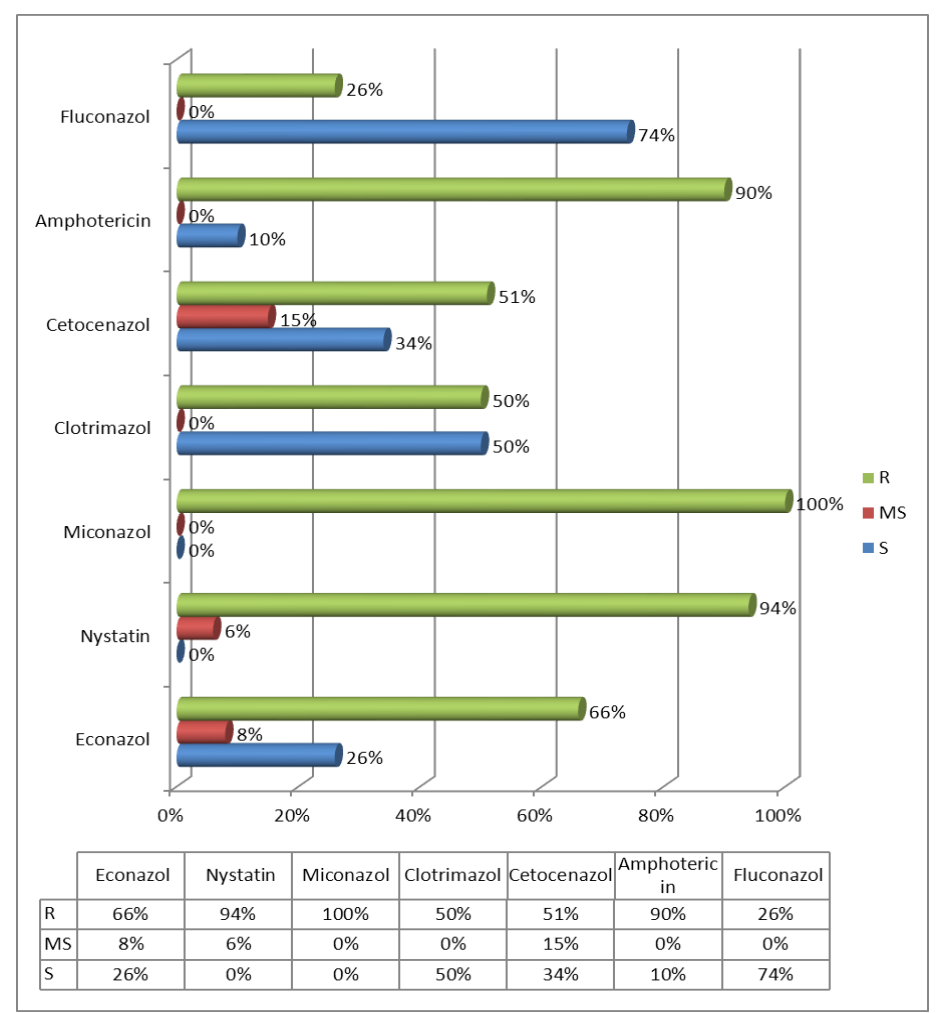

Figure 1.3 C. albicans antimycotic resistance 


\section{Literature}

[1]. Brown G.D., Denning D.W., Gow N.A.R., Netea M.G., White T. (2012): Human fungal infections: the Hidden Killers. Sci Transl Med4: 165rv113.

[2]. Gow N.A.R., van de Vërdonk F.L.Brown A.J.P., Netea M.G. (2012).Candida albicans morphogenesis and host defence: discriminating invasion from colonization. Nat. Rev.Microbiol.10,112-122.

[3]. Jacqueline M. Achkar (2010): Candida Infections of the Genitourinary Tract. Clin. Microbiol. Rev. $23(2): 253-273$.

[4]. Kanafani Z. A, Perfect J.R (2008): Resistance to Antifungal Agents: Mechanisms and Clinical Impact. Clinical Infectious Diseases. 46 (1): 120-128.

[5]. Lo H.J., Kohler J.R., DiDomenico B., Loebenberg D., Cacciapuoti A., Fink G.R. (1997): Nonfilamentous C. albicans mutants are avirulent. Cell 90: 939-949.

[6]. Peter E. Sudbery (2011): Growth of Candida albicans hyphae. Nature Reviews Microbiology 9, 737-748.

[7]. Puto K., Hila N., Shkurti R. (2013): Oral, Intestinal and Vaginal Candidiasis, Pathogenesis and Clinical Presentation in Elbasan (Albania). Indian Journal of Applied Research, Vol.III, Issue. XII Dec 2013.

[8]. Spampinato C., Leonardi D. (2013): Candida Infections, Causes, Targets, and Resistance Mechanisms: Traditional and Alternative Antifungal Agents. BioMed Research International Article ID 204237, 13 pages. 